\title{
On $\mathbb{P}$ - Hausdorff Topological Random Systems
}

\author{
Sema Kadhim Jebur \\ University of Al-Qadisiyah, College of Soil and Water Resource \\ Semh.Alisawi@qu.ed.iq \\ Recived : 17\12\2018 \\ Revised : $4 \backslash 2 \backslash 2018$ \\ Accepted : $18 \backslash 3 \backslash 2018$
}

Available online : $\quad 4 / 4 / 2018$

DOI: $10.29304 / j q c m .2018 .10 .2 .379$

\begin{abstract}
We are taking aview of topologicall random systems which is introduce considered as a mixing between two fundamental branches of mathematics "topology" and "probability Theory". The concept of $\mathbb{P}-$ Hausdorff topological random system is studied and some properties of such system are given and proved.
\end{abstract}

Key words. Random set, Topological random system, $\mathbb{P}$-neighborhood, $\mathbb{P}$-limlit point, $\mathbb{P}$-closed set and $\mathbb{P}$-Hausdorff system. 
Introduction. The "topology" and "probability Theory" (specially random sets) are the important tools in the study of pure and applied mathematics. Therefore we mix here these two theories by define the topological random system. As first step of our study are taking aview of topologicall random system and define the concept of $\mathbb{P}$ - Hausdorff topological random system. Throughout our paper we state and prove some properties of $\mathbb{P}$ - Hausdorff topological random system. This work consist of three sections. In section 2 we state the definition of random set and some concepts related with probability theory. In section 3, our new concept "the topological random system" is introduced and some concept in terms of probability concepts such as $\mathbb{P}$-neighborhood, $\mathbb{P}$-limlit point and $\mathbb{P}$-closed set are given. In Section 4 the concept of $\mathbb{P}$-Hausdorff system is introduced and some essential properties are proved.

Throughout this paper all probability space are complete (A" probability space $(\Omega, \mathcal{F}, \mathbb{P})$ is said to be complete "[1,2,3] if for every $A \in$ $\mathcal{F}$ with $\mathbb{P}(A)=0$, then $B \in \mathcal{F}$, for all subsets $B \subset A)$ and every metric space is polish space (complete separable[4]).

2. Random Sets. The origin of the recent concept of a random set energies as far back as the inspiring book by A.N. Kolmogorov [5](first published in 1933) where he arranged out the foundations of probability theory. In this section the definition of random set is given and some properties of such sets. Theset $A(\omega):=\{x \in X:(\omega, x) \in A\} \in \mathcal{B}$ is called the $\omega-\operatorname{section}$ of $A$.
Let $A: \Omega \rightarrow \mathcal{B}(X), \omega \mapsto A(\omega)$, beafunction whose values are subsets of $X$. Afunction is individually determined by its graph $\operatorname{graph}(\mathrm{A}):=\{(\omega, x) \in$ $\Omega \times \mathrm{X}: x \in A(\omega)\} \subset \Omega \times \mathrm{X}$. Conversely, each subset $A \subset \Omega \times X$ defines such a function via $\omega \mapsto A(\omega)$.

Definition 2.1[1]: "Let $(X, d)$ be a metric space which is considered a measurable space with Borel $\sigma-$ algebra $\mathcal{B}(X)$ and $(\Omega, \mathcal{F})$ be a measurable space and. The set-valued function $A: \Omega \rightarrow$ $\mathcal{B}(X), \omega \mapsto A(\omega)$, is said to be random set if for every $x \in X$ the function $\omega \mapsto d(x, A(\omega))$ is measurable. If $A(\omega)$ is closed (compact) for all $\omega \in \Omega$, it is called a random closed(compact) set".

Proposition 2.2[1]:" Let the set-valued function $\mathrm{A}: \Omega \rightarrow \mathcal{B}(X)$ take values in the subspace of closed subsets of a Polish space $X$. Then:

(i)A is a random closed set if and only if for all open sets $U \subset X$ the set $\left\{\omega: A_{\omega} \cap U \neq \emptyset\right\}$ is measurable.

(ii) If $A$ is a random closed set then $\operatorname{graph}(\mathrm{A}) \in$ $\mathcal{F} \otimes \mathcal{B}$.

The property of $A$ being a random closed set is thus slightly stronger than graph(A) being measurable and $A_{\omega}$ being closed.

For convenient, throughout this paper we adopt the following definition of random set" .

Definition $2.3[1]$ Agreement $A: \Omega \rightarrow \mathcal{B}(X)$ beaset-valued function where $X$ be "a topological space". Then A issaid to berandomclosed set if for every opensets $U \subset X$ theset $\left\{\omega: A_{\omega} \cap U \neq \varnothing\right\}$ ismeasurable. Thecomplement of randomclosedset is called randomopenset. 


\section{Examples [1]}

(a) "The set $A=\{\zeta\}$ is an RCS where $\zeta$ is a random point in" .

(b) "The set $A=(-\infty, \zeta]$ is $\operatorname{RCS}$ on $X=\mathbb{R}^{1}$ if

$\zeta$ is RV. Also the set $A=\left(-\infty, \zeta_{1}\right] \times$

$\left(-\infty, \zeta_{2}\right] \ldots \times\left(-\infty, \zeta_{n}\right]$ is $\operatorname{RCS}$ in $\mathbb{R}^{n}$ if

$\left(\zeta_{1}, \ldots, \zeta_{n}\right)$ is $n$-dimensional random vector".

\section{Theorem 2.4[1]}

(i) the closure of the complement of any closed random set is closed random set.

(ii) The closure of any random open set is closed random set.

(iii)The interior of any closed random set is open random set

(iv) the intersection of any two random set is random set.

For more detail about random set see[1] and [2].

\section{Topological Random System. In this} section the new concept of topological random system is introduced. Also the concepts of, $\mathbb{P}$-limlit point and $\mathbb{P}$-closed set are introduced.

\section{Definition 3.1 (Topological Random System)}

Let $(\Omega, \mathcal{F}, \mathbb{P})$ be a completeprobabilityspace and $(X, \tau)$ be a topologicallspace (which is considered as measurable space withBorel $\sigma-$ algebra $\mathcal{B}(X)$.) The triple $(\Omega, X, \Re)$ is called topological random system (shortly, TRS), where $\mathfrak{R}$ is the collection of random sets in $X$.

\section{Example 3.2 Agreement $(\Omega, \mathcal{F}, \mathbb{P})$ be a} completeprobabilityspace, where $\Omega:=\{H, T\}$, $\mathcal{F}:=2^{\Omega}$ and $\mathbb{P}(\{H\})=\mathbb{P}(\{T\})=\frac{1}{2}$ and let $\mathbb{R}$ be the set of all realnumbers endowed with the usualtopology( in this case $\mathbb{R}$ is polish space). Define thecollection $\Re:=\{A, B, C, D\}$ of sub sets of $\mathbb{R}$,where

$$
\begin{aligned}
& \mathrm{A}: \Omega \rightarrow \mathcal{B}(\mathbb{R}), \mathrm{A}(\omega)=\phi, \forall \omega \in \Omega \\
& \mathrm{B}: \Omega \rightarrow \mathcal{B}(\mathbb{R}), \mathrm{B}(\omega)=[0, \infty), \forall \omega \in \Omega \\
& \mathrm{C}: \Omega \rightarrow \mathcal{B}(\mathbb{R}), \mathrm{C}(\omega)=(-\infty, 0], \forall \omega \in \Omega \\
& \mathrm{D}: \Omega \rightarrow \mathcal{B}(\mathbb{R}), \mathrm{D}(\omega)=\mathbb{R}, \forall \omega \in \Omega .
\end{aligned}
$$

Thus $\mathfrak{R}:=\{A, B, C, D\}$ be the collection of randomsets in $\mathbb{R}$. (In fact is the collection of closedrandom set in $\mathbb{R})$. Hence the triple $(\Omega, \mathbb{R}, \mathfrak{R})$ is TRS.

\section{Definition 3.3(sub-topological random}

system ). The triple $(\Omega, Y, \Re)$ is said to be subtopological random system of $(\Omega, X, \Re)$ if $Y$ isasubspace (as a topologicallspace) of $X$ and the intersection of each open randomset in $X$ with $Y$ is randomopen setin $Y$.

\section{Definition 3.4 (Random Neighborhood).}

Let $(\Omega, X, \mathfrak{R})$ be an TRS and $x \in X$. A random neighborhood (shortly, RN) of $x$ is a random set $\quad N \quad$ suchthatthereexistsrandomopenset $U$ withthepropertythat

$$
\mathbb{P}\{\omega: x \in U(\omega) \subseteq N(\omega)\}=
$$

1.

The collection $\mathfrak{N}_{x}$ denoted to all Rnhd of $x$ and is called random neighborhood system (RNS) at $x$. 
Example 3.5 Consider $\mathbb{R}$ endowed with the" usual topology, and $(\Omega, \mathcal{F}, \mathbb{P})$ be any complete probability space". Let $\zeta_{\omega}, \omega \in \Omega$, be a realvaluedrandom process on $\Omega$ with continuous sample paths. Then $A=\left\{\omega: \zeta_{\omega}>0\right\}$ is RN of each elements of itself.

Theorem 3.6 The RNS $\mathfrak{N}_{x}$ has the following properties.

[RN1] If $N \in \mathfrak{N}_{x}, \rightarrow \mathbb{P}\{\omega: x \in N(\omega)\}=1$.

[RN2] If $N, M \in \mathfrak{N}_{x}, \rightarrow N \cap M \in \mathfrak{N}_{x}$.

[RN3] If $N \in \mathfrak{N}_{x}, \rightarrow \exists M \in \mathfrak{N}_{x} \ni N \in \mathfrak{N}_{y}$ for each $y \in M$.

[RN4] If $N \in \mathfrak{N}_{x}$ and $\mathbb{P}\{\omega: N(\omega) \subseteq M(\omega)\}=$ 1 , then $M \in \mathfrak{N}_{x}$.

[RN5] $G$ is random open set if and only if $G$ contains An RN of each of its points.

\section{Proof.}

[RN1]: Suppose that $N \in \mathfrak{N}_{x}$, then $\mathbb{P}\{\omega: x \in$ $U(\omega) \subseteq N(\omega)\}=1 . \quad$ Let $\quad \omega \in\{\omega: x \in$ $U(\omega) \subseteq N(\omega)\}$, then $x \in U(\omega) \subseteq N(\omega)$,i.e., $x \in N(\omega)$. Thus $\omega \in\{\omega: x \in N(\omega)\}$. Therefore $\quad\{\omega: x \in U(\omega) \subseteq N(\omega)\} \subseteq$ $\{\omega: x \in N(\omega)\}$. Since $(\Omega, \mathcal{F}, \mathbb{P})$ be a complete "probability space", then $\{\omega: x \in N(\omega)\} \in \mathcal{F}$. Now by properties of $\mathbb{P}$ we have

$$
\mathbb{P}\{\omega: x \in U(\omega) \subseteq N(\omega)\} \leq \mathbb{P}\{\omega: x \in
$$

$N(\omega)\}$

or, equivalently $1 \leq \mathbb{P}\{\omega: x \in N(\omega)\}$. Hence $\mathbb{P}\{\omega: x \in N(\omega)\}=1$.

[RN2]: Suppose that $N, M \in \mathfrak{N}_{x}$, thenthereexists two random opensets $U$ and $V$ such that

$$
\mathbb{P}\{\omega: x \in U(\omega) \subseteq N(\omega)\}=1=
$$

$\mathbb{P}\{\omega: x \in V(\omega) \subseteq M(\omega)\}$.
Clearly that

$$
\begin{aligned}
& \{\omega: x \in U(\omega) \subseteq N(\omega)\} \cap\{\omega: x \in V(\omega) \subseteq \\
& M(\omega)\} \\
& \subseteq\{\omega: x \in U(\omega) \cap V(\omega) \subseteq N(\omega) \cap M(\omega)\} . \\
& \mathbb{P}(\{\omega: x \in U(\omega) \subseteq N(\omega)\} \cap\{\omega: x \in V(\omega) \subseteq \\
& M(\omega)\})^{c} \\
& =\mathbb{P}\left(\{\omega: x \in U(\omega) \subseteq N(\omega)\}^{c} \cup\{\omega: x \in\right. \\
& \left.V(\omega) \subseteq M(\omega)\}^{c}\right) \\
& =\mathbb{P}\{\omega: x \in U(\omega) \subseteq N(\omega)\}^{c}+\mathbb{P}\{\omega: x \in \\
& V(\omega) \subseteq M(\omega)\}^{c} \\
& \quad-\mathbb{P}\left(\{\omega: x \in U(\omega) \subseteq N(\omega)\}^{c} \cap\right. \\
& \left.\{\omega: x \in V(\omega) \subseteq M(\omega)\}^{c}\right) \\
& =0+0-\mathbb{P}\left(\{\omega: x \in U(\omega) \subseteq N(\omega)\}^{c} \cap\right. \\
& \left.\{\omega: x \in V(\omega) \subseteq M(\omega)\}^{c}\right) .
\end{aligned}
$$

Thus we must have

$$
\mathbb{P}\left(\{\omega: x \in U(\omega) \subseteq N(\omega)\}^{c} \cap\right.
$$$$
\left.\{\omega: x \in V(\omega) \subseteq M(\omega)\}^{c}\right)=0 \text {, }
$$

and consequently

$$
\mathbb{P}(\{\omega: x \in U(\omega) \subseteq N(\omega)\} \cap\{\omega: x \in
$$

$V(\omega) \subseteq M(\omega)\})^{c}=0$

So, by completeness of $(\Omega, \mathcal{F}, \mathbb{P})$ we have

$$
\{\omega: x \in U(\omega) \cap V(\omega) \subseteq N(\omega) \cap
$$

$M(\omega)\}^{c} \in \mathcal{F}$.

Hence

$$
\{\omega: x \in U(\omega) \cap V(\omega) \subseteq N(\omega) \cap
$$

$M(\omega)\} \in \mathcal{F}$.

Therefore

$$
\mathbb{P}\{\omega: x \in U(\omega) \cap V(\omega) \subseteq N(\omega) \cap
$$

$M(\omega)\}=1$.

By definition of RN we get $N \cap M \in \mathfrak{N}_{x}$.

[RN3]: Suppose that $N \in \mathfrak{N}_{x}$, and take $M=\operatorname{Int}(N)$. Then for each $y \in M, y \in$ $\operatorname{Int}(N)$. Then $\mathbb{P}\{\omega: y \in \operatorname{Int}(N) \subseteq \mathrm{N}\}=1$. So $N \in \mathfrak{N}_{y}$. 
[RN4]: Suppose that $\quad N \in \mathfrak{N}_{x}$. Thenthereexistsrandomopenset $U$ withthe property that $\mathbb{P}\{\omega: x \in U(\omega) \subseteq N(\omega)\}=1$. Then $\mathbb{P}\{\omega: x \in \operatorname{Int}(N(\omega)) \subseteq N(\omega)\}=1$. If $\mathbb{P}\{\omega: N(\omega) \subseteq M(\omega)\}=1, \quad$ then $\mathbb{P}\{\omega: \operatorname{Int}(N(\omega)) \subseteq \operatorname{Int}(M(\omega))\}=1 . \quad$ So $\mathbb{P}\{\omega: x \in \operatorname{Int}(M(\omega))\}=1$ and hence $\mathbb{P}\{\omega: x \in$ $\operatorname{Int}(M(\omega)) \subseteq M(\omega)\}=1$. Therefore $M \in \mathfrak{N}_{x}$. [RN5]: If $G$ isa random openset, and $x \in G$. Since $G=\operatorname{Int}(G)$. Then $\mathbb{P}\{\omega: x \in \operatorname{Int}(G(\omega)) \subseteq$ $G(\omega)\}=1$. Hence $G \in \mathfrak{N}_{x}$. Conversely, if $G \in \mathfrak{N}_{x} \quad$ foreveryx $\in G$. Then thereexists random openset $V_{x}$ suchthat $\mathbb{P}\{\omega: x \in$ $\left.V_{x}(\omega) \subseteq G(\omega)\right\}=1$. (In fact $\left\{\omega: x \in V_{x}(\omega) \subseteq\right.$ $G(\omega)\}=\Omega)$. Hence $\bigcup_{x \in G(\omega)} \operatorname{Int}\left(V_{x}(\omega)\right)=$ $G(\omega)$. Therefore $G$ is random open set by Theorem (2.4).

Definition 3.7 ( $\mathbb{P}$-limit point). Let $(\Omega, X, \Re)$ be an TRS and $A$ be a random set in $(\Omega, X, \Re)$. Apoint $x \in X$ issaidtobe $\mathbb{P}$-limit point of $A$ if $\mathbb{P}\{\omega:[N(\omega)-\{x\}] \cap A(\omega) \neq$ $\emptyset\}=1$ for every RN $N$ of $x$.

Thesetof all $\mathbb{P}$-limitpoint of $A$ iscalledthe $\mathbb{P}$-derivedset and is denotedby $\mathbb{P}-D(A)$.

Example 3.8 Consider the TRS $(\Omega, \mathbb{R}, \Re)$ given in Example 3.2 with $=(-1, \infty)$. Then -1 is $\mathbb{P}$-limit point of .

Definition 3.9 ( $\mathbb{P}$-closed set) The (deterministic) subset $A$ of $X$ is said tobe $\mathbb{P}$-closedset if ithas all of its $\mathbb{P}$-limit points points. That is $A$ of $X$ is $\mathbb{P}$-closedset if and only if $\mathbb{P}-D(A) \subseteq A$. Thecomplement of $\mathbb{P}$-closedset is called $\mathbb{P}$ - openset.
Example 3.10 Consider Example 3.8. Then all intervals of the form $[a, \infty) \subseteq \mathbb{R}$ are $\mathbb{P}$-closed.

Note 3.11. The set $G$ is $\mathbb{P}$-open set if and only if $\mathbb{P}-D(G) \cap G=\emptyset$.

Lemma 3.12 The finite union of all $\mathbb{P}$-closedsets is $\mathbb{P}$-closedset.

Proof. Assume that $\left\{A_{i}: i=1,2, \ldots, n\right\}$ bea collectionof $\mathbb{P}$-closed sets. To showthat $A=\bigcup_{i=1}^{n} A_{i}$ isa $\mathbb{P}$-closedset. Let $x \in X$ bea $\mathbb{P}$-limitpoint of theset $A$. Then for every $\mathrm{RN}$ $N$ of $x$ wehave $\quad \mathbb{P}\{\omega:[N(\omega)-\{x\}] \cap$ $A(\omega) \neq \emptyset\}=1$. Set $F=\{\omega:[N(\omega)-$ $\{x\}] \cap A(\omega) \neq \emptyset\} \quad$ with $\quad \mathbb{P}(F)=1$. Hence $[N(\omega)-\{x\}] \cap A(\omega) \neq \varnothing, \quad \forall \omega \in F . \quad$ Then $[N(\omega)-\{x\}] \cap \bigcup_{i=1}^{n} A_{i}(\omega) \neq \varnothing, \quad \forall \omega \in F$,or equivalently $\bigcup_{i=1}^{n}\left\{[N(\omega)-\{x\}] \cap A_{i}(\omega)\right\} \neq$ $\emptyset, \forall \omega \in F$. Then there exists $i_{0} \in\{1,2, \ldots, n\}$ suchthat $\quad[N(\omega)-\{x\}] \cap A_{i_{0}}(\omega) \neq \varnothing$, $\forall \omega \in F$. That is $\quad \mathbb{P}\{\omega:[N(\omega)-\{x\}] \cap$ $\left.A_{i_{0}}(\omega) \neq \emptyset\right\}=1$. Then $x$ is a $\mathbb{P}$-limit point of $A_{i_{0}}$. But $A_{i_{0}}$ is a $\mathbb{P}$-closed set, then $x \in A_{i_{0}}$. Consequently $x \in \bigcup_{i=1}^{n} A_{i}=A$. Since $x$ is an arbitrary it follows that $\bigcup_{i=1}^{n} A_{i}=A$ contains all of its $\mathbb{P}$-limit points. Hence $\bigcup_{i=1}^{n} A_{i}=A$ is $\mathbb{P}-$ closed set.

4. $\mathbb{P}$-Hausdorff system. In this final section the concept of $\mathbb{P}$-Hausdorff system is introduced and studied. The " Hausdorff property" is one of the important properties in the study the topology and its applications. Therefore we focus our study to study this concept in terms of probability theory and random set. 
Definition 4.1 A topological random system $(\Omega, X, \Re)$ is said to be $\mathbb{P}-$ Hausdorff $(\mathbb{P}-$ $T_{2}$ ) if for every there exist two distinctpoints $x, y \in X$, tworandom opensets $A$ and $B$ in $X$ such that $x \in A, \quad y \in B$ andP $\{\omega: A(\omega) \cap$ $B(\omega) \neq \emptyset\}=0$ or equivalently $\mathbb{P}\{\omega: A(\omega) \cap$ $B(\omega)=\emptyset\}=1$.

Example 4.1 Let $(\Omega, \mathcal{F}, \mathbb{P})$ be a complete probabilityspace, where $\Omega:=\{H, T\}, \quad \mathcal{F}:=2^{\Omega}$ and $\mathbb{P}(\{H\})=\mathbb{P}(\{T\})=\frac{1}{2}$ and let $\mathbb{R}$ be the set of all real numbers endowed with the usualtopology. Define the collection $\mathfrak{R}:=\left\{A_{x}: x \in \mathbb{R}\right\}$ of sub sets of $\mathbb{R}$, where

$$
A_{x}: \Omega \rightarrow \mathcal{B}(\mathbb{R}), A_{x}(\omega)=\{x\}, \forall \omega \in \Omega .
$$

Thus $\mathfrak{R}:=\left\{A_{x}: x \in \mathbb{R}\right\}$ be the collection of randomsets in $\mathbb{R}$. Hence the triple $(\Omega, \mathbb{R}, \mathfrak{R})$ is TRS. Since $\mathbb{P}\{\omega: A(\omega) \cap B(\omega)=\emptyset\}=$ $\mathbb{P}(\Omega)=1$. Then $(\Omega, \mathbb{R}, \mathfrak{R})$ is $\mathbb{P}-T_{2}$ space.

Theorem 4.3 The subspace of a $\mathbb{P}-T_{2}$ spaceis $\mathbb{P}-T_{2}$.

Proof: $\operatorname{Let}(\Omega, X, \Re)$ be atopological random system and $\left(Y, \tau_{Y}\right)$ be asubspace $\operatorname{of}(X, \tau)$. Let $x, y \in Y \quad$ with $x \neq y . \quad$ Then $x, y \in X$. Byhypothesis, thereexisttwo open randomsets Aand $B$ in $X$ suchthat $x \in A, y \in B$ andP $\{\omega: A(\omega) \cap B(\omega) \neq \varnothing\}=0 . \quad$ Define $C(\omega):=A(\omega) \cap Y$ and $D(\omega):=B(\omega) \cap Y$ are two random sets in $Y$ with $x \in C(\omega)$ and $y \in D(\omega)$. Let $F=\{\omega: A(\omega) \cap B(\omega) \neq \varnothing\}$, such that $\mathbb{P}(F)=0$. Then $\{\omega: C(\omega) \cap$ $D(\omega) \neq \emptyset\} \subseteq F . \quad$ By completeness of $(\Omega, \mathcal{F}, \mathbb{P})$ we have $\{\omega: C(\omega) \cap D(\omega) \neq \emptyset\} \in \mathcal{F}$. Thus $\mathbb{P}\{\omega: C(\omega) \cap D(\omega) \neq \emptyset\} \leq$ $\mathbb{P}\{\omega: A(\omega) \cap B(\omega) \neq \emptyset\}=0$. It follows from completenessof theprobability spacethat $\mathbb{P}\{\omega: C(\omega) \cap D(\omega) \neq \emptyset\}=0$. Thus the subsystem $(\Omega, Y, \Re)$ is $\mathbb{P}-T_{2}$.
Theorem 4.4 Every singletonset in a $\mathbb{P}-T_{2}$ space is $\mathbb{P}$-closedset.

Proof. Let $(\Omega, X, \Re)$ be a $\mathbb{P}-T_{2}$ and let $x \in X$. Toshow that $\{x\}$ is $\mathbb{P}$-closed set. Let $y \in X$, with $x \neq y$. To prove that $y$ is not $\mathbb{P}-$ limit point of $\{x\}$. i.e., thereexists RN Nof ysuch that $\mathbb{P}\{\omega:[N(\omega)-\{y\}] \cap\{x\}=\emptyset\}=$ 1. Since $(\Omega, X, \mathfrak{R})$ is $\mathbb{P}-T_{2}$, thereexist tworandom opensets $A$ and $B$ in $X$ suchthat $x \in$ $M, y \in N$ andP $\{\omega: M(\omega) \cap N(\omega)=\emptyset\}=1$. Set $\quad F=\{\omega: M(\omega) \cap N(\omega)=\emptyset\}$, with $\mathbb{P}(F)=1$. Then $M(\omega) \cap N(\omega)=\emptyset, \forall \omega \in F$. Then $x \notin N(\omega), \quad \forall \omega \in F$. That is $\mathbb{P}\{\omega: x \notin$ $N(\omega)\}=1 \quad \operatorname{or} \mathbb{P}\{\omega:\{x\} \cap N(\omega)=\emptyset\}=1$. Hence $\mathbb{P}\{\omega:\{x\} \cap(N(\omega)-\{y\})=\emptyset\}=1$.

Consequently $\{x\}$ is $\mathbb{P}$-closed set.

Corollary 4.5 A finite (deterministic) sub set of a $\mathbb{P}-T_{2}$ is $\mathbb{P}$-closed set.

Proof. Thisfollows fromTheorem 4.4andLemma3.12.

Theorem 4.6 The $\operatorname{RS}(\Omega, X, \Re)$ is $\mathbb{P}-T_{2}$ $\leftrightarrow$ foreach pair $x, y \in X$, thereexists aP - nhd $N_{y}$ of $y$ suchthat $\mathbb{P}\left\{\omega: x \notin \overline{N_{y}}(\omega)\right\}=1$.

Proof: $\quad \operatorname{Supposing}(\Omega, X, \mathfrak{R})$ is $\mathbb{P}-T_{2}$ and let $x, y \in X$ with $x \neq y$. Then thereexist two randomopen sets $G$ and $H$ in $X$ suchthat $x \in G$, $y \in H \operatorname{and} \mathbb{P}\{\omega: G(\omega) \cap H(\omega)=\emptyset\}=1$.

$\operatorname{Then} \mathbb{P}\{\omega: y \in H(\omega) \subseteq X-G(\omega)\}=1$. Then $X-G(\omega)$ is closed $\mathbb{P}-$ nhd of $y$ and $\mathbb{P}\{\omega: x \notin$ $X-G(\omega)\}=1 . \quad \operatorname{Set}_{y}=X-G(\omega), \quad$ then $\overline{N_{y}}=N_{y}$, so $N_{y}$ is $\mathbb{P}-$ nhd and $\mathbb{P}\{\omega: x \notin$ $\left.\overline{N_{y}}(\omega)\right\}=1$ 
Conversely, suppose that for each pair $x, y \in X$,there exists a $\mathbb{P}-$ nhd $\quad N_{y}$ of $y$ suchthat $\mathbb{P}\left\{\omega: x \notin \overline{N_{y}}(\omega)\right\}=1$. Since $\overline{N_{y}} \supseteq N_{y}$, then by Theorem (3.6) RN4 $\overline{N_{y}}$ is $\mathbb{P}-$ nhd of $y$. Since $\overline{N_{y}}$ is closed random set, then $X-\overline{N_{y}}$ is open random set with $x \in X-$ $\overline{N_{y}}$ and $y \notin X-\overline{N_{y}}$. Put $-\overline{N_{y}}=N_{x}$, we see that there is a $\mathbb{P}-$ nhd $N_{x}$ of $x$ and a $\mathbb{P}$-nhd $\overline{N_{y}}$ of $y$ such that $\mathbb{P}\left\{\omega: N_{x}(\omega) \cap \overline{N_{y}}(\omega)=\right.$ $\emptyset\}=1$. Consequently $\mathbb{P}\left\{\omega: N_{x}(\omega) \cap N_{y}(\omega)=\right.$ $\emptyset\}=1$. Therefore $(\Omega, X, \Re)$ is $\mathbb{P}-T_{2}$.

Theorem 4.7 The $\operatorname{RS}(\Omega, X, \Re)$ is $\mathbb{P}-T_{2}$ if and only if for every collection $\left\{F_{\lambda}: \lambda \in \Lambda\right\}$ of closed $\mathbb{P}-$ nhd ofeach $x \in X$ we have $\mathbb{P}\left\{\omega: \bigcap_{\lambda \in \Lambda} F_{\lambda}(\omega)=\{x\}\right\}=1$.

Proof. Suppose that $(\Omega, X, \mathfrak{R})$ is aP $-T_{2}$ RS. Let $x, y \in X \quad$ with $x \neq y$. Then thereexist $G, H \in R O S$ suchthat $x \in G, y \in H$ and $\mathbb{P}\{\omega: G(\omega) \cap H(\omega)=\emptyset\}=$ 1. $\operatorname{Thus} \mathbb{P}\{\omega: G(\omega) \subseteq X-H(\omega)\}=1$. Hence $X-H(\omega)$ is a closed $\mathbb{P}-$ nhd of $x$ and by completeness of $(\Omega, \mathcal{F}, \mathbb{P})$ we have $\mathbb{P}\{\omega: y \in X-H(\omega)\}=0$. If $\left\{F_{\lambda}: \lambda \in \Lambda\right\}$ of closed $\mathbb{P}-$ nhd of each $x \in X$, then $\mathbb{P}\{\omega: y \in$ $\left.\bigcap_{\lambda \in \Lambda} F_{\lambda}(\omega)\right\}=0$. Since $y$ is an arbitrary, then $\mathbb{P}\left\{\omega: \bigcap_{\lambda \in \Lambda} F_{\lambda}(\omega)=\{x\}\right\}=1$.

Conversely, suppose that $\mathbb{P}\left\{\omega: \bigcap_{\lambda \in \Lambda} F_{\lambda}(\omega)=\right.$ $\{x\}\}=1$ for every collection $\left\{F_{\lambda}: \lambda \in \Lambda\right\}$ of closed $\mathbb{P}-$ nhd of $\operatorname{each} x \in X$. Lety $\in$ $X$ with $x \neq y$. Since $\mathbb{P}\left\{\omega: y \in \bigcap_{\lambda \in \Lambda} F_{\lambda}(\omega)\right\}=$ 0 , then there exists closed $\mathbb{P}-$ nhd $N$ of each $x$ $\mathbb{P}\{\omega: y \notin N(\omega)\}=1$. Thenthere exists $G \in$ $R O S$ such that $\mathbb{P}\{\omega: x \in G(\omega) \subseteq N(\omega)\}=1$.
Therefore $G, N^{c} \in R O S$ such that $x \in G$ and $y \in N^{c}$. Finally, we must show that $F=$ $\left\{\omega: G\left((\omega) \cap N^{c}(\omega)=\emptyset\right\} \in \mathcal{F}\right.$, and $\mathbb{P}(F)=$ 1.

We $\quad$ have $F=\left\{\omega: G(\omega) \cap N^{c}(\omega)=\emptyset\right\}=$ $\{\omega: G(\omega) \subseteq N(\omega)\}$

$\{\omega: x \in G(\omega) \subseteq N(\omega)\}$.

By completeness of $(\Omega, \mathcal{F}, \mathbb{P}), \quad F \in$ $\mathcal{F}$ and $\mathbb{P}(F)=1$. This means that $(\Omega, X, \mathfrak{R})$ is a $\mathbb{P}-T_{2}$ RS.

Corollary 4.8 The $\operatorname{RS}(\Omega, X, \mathfrak{R})$ is $\mathbb{P}-T_{2}$ if and only if for every collection $\left\{N_{\lambda}: \lambda \in \Lambda\right\}$ of $\mathbb{P}-\quad$ nhd $\quad$ of $\quad \operatorname{each} x \in X \quad$ we have $\mathbb{P}\left\{\omega: \bigcap_{\lambda \in \Lambda} \overline{N_{\lambda}}(\omega)=\{x\}\right\}=1$.

Proof Since the closure of random set is closed random set (by Theorem 2.4 ) then the result followed directly from Theorem 4.6.

Theorem 4.9 The $\operatorname{RS}(\Omega, X, \Re)$ is $\mathbb{P}-T_{2}$ if and only if for every finite (deterministic) subset $\left\{x_{i}: i=1,2, \ldots, n\right\}$ of $X$ there exists $\mathrm{RN}$ $N_{i}$ of $x_{i}$ for every $i=1,2, \ldots, n$ such that for every $i, j=1,2, \ldots, n$ with $i \neq j \quad$ we have $\mathbb{P}\left\{\omega: N_{i}(\omega) \cap N_{j}(\omega)=\emptyset\right\}=1$.

Proof. Supposing that $(\Omega, X, \Re)$ be aP $-T_{2}$ and $\left\{x_{i}: i=1,2, \ldots, n\right\} \subseteq X \quad$ with $\quad x_{i} \neq x_{j}$, $\forall i, j=1,2, \ldots, n$ with $i \neq j$. By hypothesis there exist $N_{i j}, N_{j i} \in R O S$ such that $x_{i} \in N_{i j}, x_{j} \in$ $N_{j i}$ and $\mathbb{P}\left\{\omega: N_{i j}(\omega) \cap N_{j i}(\omega)=\emptyset\right\}=1$. Let $N_{i}(\omega)=\cap\left\{N_{i j}(\omega): j=1,2, \ldots, n, i \neq j\right\}$. 
Then by Theorem $2.4 N_{i}(\omega) \in R O S$, for every $i=1,2, \ldots, n$. To show that $\mathbb{P}\left\{\omega: N_{i}(\omega) \cap\right.$ $\left.N_{j}(\omega)=\emptyset\right\}=1, \quad$ for $\quad$ every $i, j=1,2, \ldots, n$ with $i \neq j . \quad \operatorname{Set} F=\left\{\omega: N_{i}(\omega) \cap N_{j}(\omega)=\emptyset\right\}$, for every $i, j=1,2, \ldots, n$ with $i \neq j$. Let $i, j=$ $1,2, \ldots, n$ with $i \neq j$. Then $\forall \omega \in F$ we have

$N_{i}(\omega) \cap N_{j}(\omega)=\left(\bigcap_{i \neq j} N_{i j}(\omega)\right) \cap$

$\left(\bigcap_{i \neq j} N_{j i}(\omega)\right)$

$$
=\bigcap_{i \neq j}\left(N_{i j}(\omega) \cap N_{j i}(\omega)\right)=\emptyset .
$$

Then $F=\left\{\omega: N_{i j}(\omega) \cap N_{j i}(\omega)=\emptyset\right\}$ and hence $\mathbb{P}(F)=1$.

Conversely, suppose that for every finite (deterministic) subset $\left\{x_{i}: i=1,2, \ldots, n\right\}$ of $X$ there exists RN $N_{i}$ of $x_{i}$ for every $i=1,2, \ldots, n$ such that forevery $i, j=1,2, \ldots, n$ with $i \neq$ jwehave

$$
\mathbb{P}\left\{\omega: N_{i}(\omega) \cap N_{j}(\omega)=\right.
$$

$\emptyset\}=1$. It follows in particular that forany twodistinct points $x, y$ there exist $M, N \in$ ROS such that $x \in N, y \in M$ and $\mathbb{P}\{\omega: M(\omega) \cap$ $N(\omega)=\emptyset\}=1$. Thus $(\Omega, X, \Re)$ be a $\mathbb{P}-T_{2}$.

Theorem 4.10 Let $(\Omega, X, \Re)$ be an RS. If each point of $X$ admits a $\tau$-closed $\mathbb{P}$-nhd of $x$ which is a $\mathbb{P}-T_{2}$ sub-system of $(\Omega, X, \Re)$, then $(\Omega, X, \mathfrak{R})$ is $\mathbb{P}-T_{2}$.
Proof. Let $x \in X$ and let $Y$ be a $\tau-\operatorname{closedP}-$ nhd of $x$ in $X$. Such that $(\Omega, Y, \mathfrak{R})$ is $\mathbb{P}-T_{2}$ sub-system of $(\Omega, X, \Re)$. First we need to show that every $\tau_{Y}$-closed $\mathbb{P}-$ nhd of $x$ is a $\tau$-closed $\mathbb{P}$-nhd of $x . \quad N^{*}$ be a $\tau_{Y}-\operatorname{closed} \mathbb{P}-$ nhd of $x$. Then thereis a $\tau-$ closedP - nhd $N$ of $x$ such that $N^{*}=N \cap$ $Y$. Since $N$ and $Y$ are random $\tau$-closed sets then by Theorem $2.4 N^{*}=N \cap Y$ is random $\tau$-closed set and so $N^{*}$ is $\tau$-closed $\mathbb{P}-$ nhd of $x$. Now, let $\left\{F_{\lambda}: \lambda \in \Lambda\right\}$ be a collection of $\tau_{Y}$-closed $\mathbb{P}-$ nhd of $x$ by hypothesis we have $\mathbb{P}\left\{\omega: \bigcap_{\lambda \in \Lambda} F_{\lambda}(\omega)=\{x\}\right\}=1$. It follows that $\left\{F_{\lambda}: \lambda \in \Lambda\right\}$ be a collection of $\tau-$-closed $\mathbb{P}-$ nhd of $x$. Consequently, $(\Omega, X, \Re)$ is $\mathbb{P}-T_{2}$.

\section{References :}

[1] I. Molchanov, "Theory of Random Sets" Springer, London, (2005).

[2] Hung T. Nguyen," An Introduction to Random Sets", Taylor \& Francis Group, LLC(2006).

[3] R.B. Ash,"Real Analysis and Probability", Academic Press,Inc. New York. (1972).

[4] S. Willard," General Topology", AddisonWesty Pub.co.,Inc.(1970).

[5] A. N. Kolmogorov, "Foundations of the Theory of Probability". Chelsea, New York (1950). 


$$
\begin{aligned}
& \text { حول النظم التبولوجية العشوائية الهاوزدورفية من النمط P } \\
& \text { سمه كاظم جبر } \\
& \text { جامعة القادسية/كلية الزراعة /قسم علوم التربة و الموارد المائية }
\end{aligned}
$$

\section{Semh.Alisawi@qu.ed.iq}

المستخلص:

قدمنا في هذا البحث مفهوم النظام التبولوجي العشو ائي الذي يعتبر كخليط بين فرعين اساسيين من فروع

الرياضيات " التبولوجيا" و "نظرية الاحتمال". تم دراسة النظم التبولوجية العشوائية الهاوزدورفية من النمط و تم تقديم بعض خو اص هذا النظام مع بر هانها. 